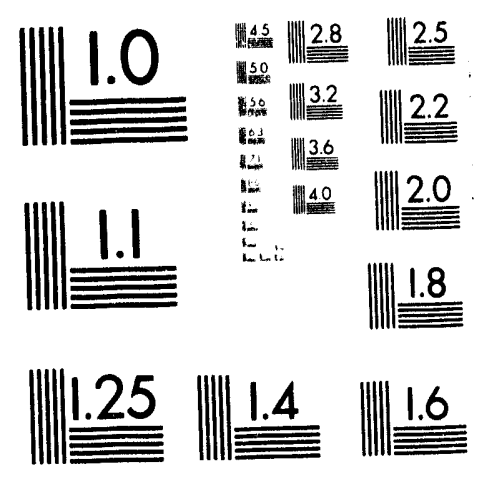



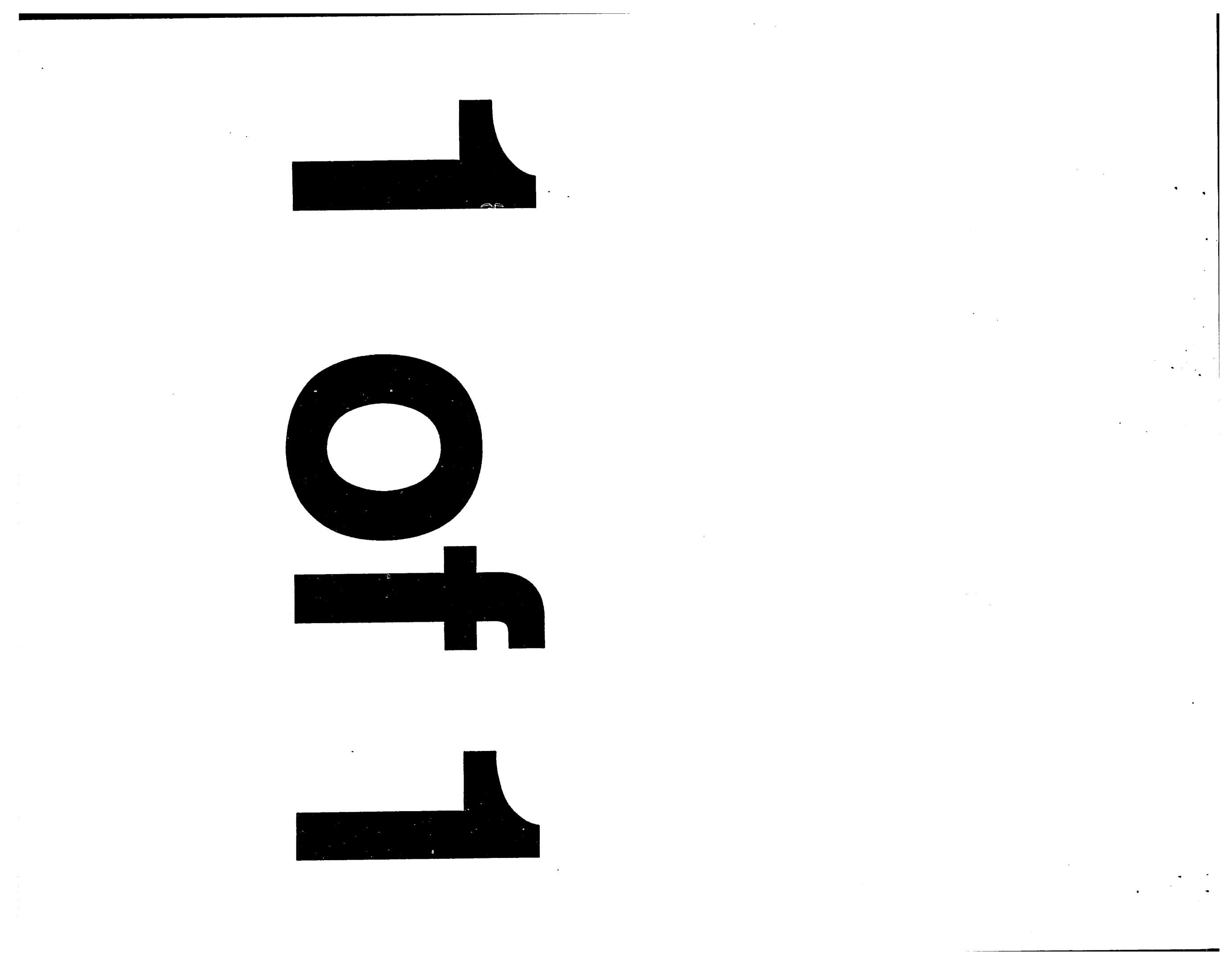


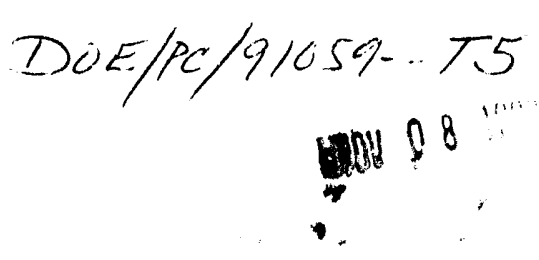

\section{BIOLOGICAL UPGRADING OF COAL LIQUIDS QUARTERLY REPORT}

July 1, 1993 - September 30, 1993

Contractor: Engineering Resources, Inc.

Contract No. DE-AC22-91PC91059

Contract Duration: September 24, 1991 - September 24, 1994

\section{SUMMARY}

Denitrifying and sulfate reducing bacteria are being developed as additional isolates capable of removing sulfur and nitrogen from coal liquids and the model compound naphtha. Both types of bacteria are anaerobic, which during heteroatom removal, should result in less destruction of the carbon in the coal liquids. Denitrifying bacteria grow on alternative carbon sources and should use the sulfur in coal liquids as their sole source of nitrogen. It is estimated that the bacterial cells contain approximately 1 percent sulfur and 15 percent nitrogen. Thus, it seems feasible to use these anaerobic bacteria to incorporate heteroatom compounds into cell mass. This report summarizes the results of isolation, growth and heteroatom utilization experiments on coal liquids and the model compound naphtha.

\section{INTRODUCTION}

The presence of heteroatom and aromatic compounds in liquids obtained from coal liquefaction processes requires expensive hydrogenation treatment to derive an acceptable liquid fuel. This project will develop a simple biological process for removing $\mathrm{N}, \mathrm{O}$ and $\mathrm{S}$ and reducing the aromaticity of coal liquids. Microorganisms, employing biocatalysts, are known to degrade aromatic heteroatom compounds in nature to $\mathrm{NH}_{3}, \mathrm{SO}_{4}=$ and $\mathrm{CO}_{2}$. Preliminary experiments in the ERI laboratories to determine the feasibility of biological removal of $\mathrm{N}, \mathrm{O}$ and $\mathrm{S}$ from coal and shale oil liquids have shown up to 20 percent nitrogen removal, 40 percent sulfur removal and 100 percent oxygen removal in a simple one stage incubation.

A biological process for upgrading of coal liquids would offer significant advantages, such as operation at ordinary temperature and pressure with better energy efficiency. Of greater importance is the fact that microorganisms do not require an external supply of hydrogen for heteroatom removal, obtaining the required hydrogen from water. Furthermore, the biocatalysts are continuously regenerated by growth on the heteroatom compounds. Ring structures are degraded as the heteroatoms are removed. The heteroatoms are in an innocuous form, such as $\mathrm{NH}_{3}, \mathrm{SO}_{4}=, \mathrm{CO}_{2}$ and $\mathrm{H}_{2} \mathrm{O}$. Therefore, there is significant potential for the development of an economical biological process for upgrading coal liquids.

This project will screen known bacteria and develop isolates for $\mathrm{N}, \mathrm{O}$ and $\mathrm{S}$ removal and aromaticity reduction. The performance of the best of these cultures will be optimized for complete heteroatom removal in a single step. Continuous reactor experiments will be conducted with the optimal cultures to determine reaction kinetics and reactor design. The design and economics of this process, including product recovery, will be projected to define economic feasibility and high cost areas.

\section{MASTER}




\section{PURPOSE}

The purpose of this report is to present the results of batch culture selection and continuous stirred tank reactor studies for the removal of heteroatom compounds from coal liquids and the model compound naphtha. Denitrifying and sulfate-reducing bacteria are being utilized to incorporate heteroatom sulfur and nitrogen into cell mass under anaerobic conditions.

\section{RESEARCH ACTIVITY}

\section{TASKS 2/3. CULTURE SCREENING AND ISOLATION/CULTURE OPTIMIZATION}

\section{Utilization of Denitrifying Bacteria for Sulfur Removal}

The first step in obtaining enrichments of denitrifying bacteria for sulfur removal was to inoculate a consortia of bacteria on various carbon sources in the presence of either naphtha or coal liquid as the sulfur source. A mixture of sludge, soil and petroleum waste was inoculated into a mixture of carbon sources and either naphtha or coal liquid as the source of sulfur. Both heavy straight run naphtha and full range cracked naphtha were used along with Wilsonville coal liquid $\mathrm{E}$.

Table 1 shows the enrichment history on the naphtha model compounds. In this and all other tables, $\mathrm{H}$ denotes full range cracked naphtha and $\mathrm{L}$ denotes heavy straight run naphtha. In following Table 1 , the consortium was first inoculated into a bottle containing 5 percent full range cracked naphtha and $1 \mathrm{~g} / \mathrm{L}$ each of nine potential carbon sources. A relatively high optical density (representing cell growth) was obtained. One to two drops of the resulting culture enrichment were then inoculated into bottles containing 5 percent naphtha and $5 \mathrm{~g} / \mathrm{L}$ of one of the carbon sources. This procedure ensured that increases in optical density would be due to growth on the carbon source and naphtha instead of inoculum carryover. The procedure was repeated for eight transfers using the best carbon sources and either full range cracked or heavy straight run naphtha. Lactate and perhaps glycerol appeared to be suitable carbon sources for the rapid growth of the denitrifying bacteria. A similar procedure was designed to utilize heavy straight run naphtha (Table 2) or water extracted naphtha (Table 3) as the source of sulfur.

Table 4 shows the results of a similar experiment using coal liquid $\mathrm{E}$ as the sulfur source. Very good growth occurred when using glycerol as the carbon source through four transfers. However, the resulting sulfur uptake from the coal liquid was not appreciable in $312 \mathrm{hr}$, when using acetate as the carbon source (see Table 5).

In the next set of experiments, full range cracked naphtha was treated with an enrichment of denitrifying bacteria as a sole sulfur source. Whenever the culture grew to a relatively high optical density, the naphtha was extracted and added to fresh medium inoculated with the same culture. Continued growth indicated that the sulfur in naphtha had to be used as the sulfur source. It was expected that less growth would occur after the first few transfers, since the more tightly bound sulfur in the naphtha would become limiting. The results from the experiment are shown in Table 6.

From Table 6, it appears that after the sixth transfer, the culture started to grow better. This indicates that the culture adapted to naphtha and was capable of utilizing additional sulfur from the naphtha. Precipitation of $\mathrm{FeS}$ may be indicated by the appearance of finely disseminated black flakes in the vessel. 
The naphtha was subjected to elemental analysis of sulfur. However, the analysis showed no significant drop in the sulfur content. This result could be due to (1) low sulfur content in bacterial mass; (2) the consumption of both sulfur and hydrocarbons in naphtha; (3) errors due to elemental analysis.

Several vendors have been contacted regarding the use of elemental analysis for sulfur analysis. Each of the vendors indicated that this technique is unsuitable for sulfur concentrations of $1000 \mathrm{ppm}$ or below, the typical levels in coal liquids and naphtha. Other methods of analysis are being considered which can yield meaningful data at these sulfur concentrations.

In another set of experiments, a mixture of denitrifying bacteria was enriched with heavy straight run naphtha. This enrichment grew at a rapid rate with the naphtha as the sole sulfur source (see Table 7).

A GC-MS analysis of the two naphtha sources was prepared by an independent laboratory. In comparing the two sources, it was found that the full range cracked naphtha contains a variety of identifiable sulfur compounds including thiophenes and benzothiophenes. Heavy straight run naphtha, on the other hand, contained almost no identifiable sulfur compounds (see attachment).

\section{Utilization of Sulfate Reducing Bacteria for Nitrogen Removal}

Similar procedures are being utilized for nitrogen removal from naphtha and coal liquids by employing sulfate reducing bacteria. These bacteria grow slower than denitrifying bacteria, but also incorporate a larger percentage of the heteroatom compound into cell mass. Tables 8 and 9 present results of the enrichment histories of the sulfate reducing bacteria on various carbon sources and naphtha or coal liquid E, respectively. As is noted in Table 8, pyruvate, fructose and glucose appear to be promising carbon sources for nitrogen uptake from naphtha. Experimental results with coal liquid $E$ will be apparent in a few weeks.

The heavy straight run naphtha has also been treated with sulfate-reducing bacteria to test bacterial growth after several transfers. No bacterial growth was detected with either naphtha or coal liquids as the nitrogen source.

Sulfate-reducing bacteria were employed in an attempt to remove heteroatom nitrogen from coal liquids. These bacteria were supplied sulfur, but depend upon the coal liquid as their sole source of nitrogen. Experiments have begun using various carbon sources for these sludge isolates. As is noted in Tables 10 and 11, no growth has occurred yet under anaerobic or aerobic conditions.

\section{Enrichments of Quinoline, Benzoquinoline, Indole and Carbazole-Consuming Cultures}

Enrichments were developed on quinoline, benzoquinoline, indole and carbazole. These sulfur compounds were found to be present in GCMS analyses of naphtha and coal liquids. Experiments were performed with these enrichments at $\mathrm{pH} 6.6$, a temperature of $37^{\circ} \mathrm{C}$, aerobic conditions and at $150 \mathrm{rpm}$. Inoculation was from a mixture of sludge and chemical waste samples in nitrogen-limited medium. Eight test cases were inoculated with naphtha using different carbon and nitrogen sources. 

a. quinoline $(1 \mathrm{~g} /)$
b. quinoline $(1 \mathrm{~g} /)+$ glucose $(2 \mathrm{~g} /)$
c. benzoquinoline $(1 \mathrm{~g} /)$
d. benzoquinoline $(1 \mathrm{~g} /)+$ glucose $(2 \mathrm{~g} /)$
e. indole $(1 \mathrm{~g} /)$
f. indole $(1 \mathrm{~g} /)+$ glucose $(2 \mathrm{~g} /)$
g. carbazole $(1 \mathrm{~g} /)$
h. carbazole $(1 \mathrm{~g} / \mathrm{l})+$ glucose $(2 \mathrm{~g} /)$

Consumption of oxygen was detected as an indication of growth. After incubation for seven days, treatments $\mathrm{a}, \mathrm{g}$ and $\mathrm{h}$ began to consume oxygen. Since the medium is nitrogen limited, bacterial growth is dependent upon the utilization of heteroatom nitrogen. These experiments will continue.

\section{TASK 4. CSTR EXPERIMENTS}

Experiments are also in progress using denitrifying and sulfate reducing bacteria for anaerobic sulfur and nitrogen removal in continuous culture. Gas wash bottles have been modified for anaerobic use, and serve as the CSTRs in these experiments.

\section{DISCLAIMER}

This report was prepared as an account of work sponsored by an agency of the United States Government. Neither the United States Go:ernment nor any agency thereof, nor any of their employees, makes any warranty, express or implied, or assumes any legal liability or responsibility for the accuracy, completeness, or usefulness of any information, apparatus, product, or process disclosed, or represents that its use would not infringe privately owned rights. Reference herein to any specific commercial product, process, or service by trade name, trademark, manufacturer, or otherwise does not necessarily constitute or imply its endorsement, recommendation, or favoring by the United States Government or any agency thereof. The views and opinions of authors expressed herein do not necessarily state or reflect those of the United States Government or any agency thereof. 
Table 1. Enrichment of Denitrifying Bacteria on Various Carbon Sources Using Naphtha as the Sulfur Source

\begin{tabular}{|c|c|c|c|c|c|}
\hline Transfer & Amount of Inoculum & Bottle & Carbon Source & $\begin{array}{l}\text { Sulfur } \\
\text { Source }\end{array}$ & $\begin{array}{l}\text { OD } \\
600 \mathrm{~nm}\end{array}$ \\
\hline $\begin{array}{l}\text { First } \\
7 / 9\end{array}$ & $\begin{array}{l}5 \mathrm{~mL} \text { of mixture of } \\
\text { sludge, soil and } \\
\text { petroleum waste }\end{array}$ & $\mathrm{A}$ & $\begin{array}{l}\text { Acetate, Pyruvate, } \\
\text { Glucose, Fructose, } \\
\text { Methanol, Lactate, } \\
\text { Succinate, Ethanol, } \\
\text { Glycerol }(1 \mathrm{~g} / \mathrm{L} \text { each })\end{array}$ & $\begin{array}{l}\text { Naphtha } \\
(\mathrm{H}, 5 \%)\end{array}$ & 0.576 \\
\hline \multirow{9}{*}{$\begin{array}{l}\text { Second } \\
7 / 12\end{array}$} & \multirow{9}{*}{$1-2$ drops in $50 \mathrm{~mL}$} & $\mathrm{Z1}$ & Acetate $(5 \mathrm{~g} / \mathrm{L})$ & \multirow{9}{*}{$\begin{array}{l}\text { Naphtha } \\
(\mathrm{H}, 5 \%)\end{array}$} & 0.245 \\
\hline & & $\mathrm{Z3}$ & Pyruvate $(5 \mathrm{~g} / \mathrm{L})$ & & 0.359 \\
\hline & & Z5 & Glucose $(5 \mathrm{~g} / \mathrm{L})$ & & 0.724 \\
\hline & & $\mathrm{Z7}$ & Fructose $(5 \mathrm{~g} / \mathrm{L})$ & & 0.661 \\
\hline & & Z9 & Methanol $(5 \mathrm{~g} / \mathrm{L})$ & & 0.045 \\
\hline & & $\mathrm{Z} 11$ & Lactate $(5 \mathrm{~g} / \mathrm{L})$ & & 0.385 \\
\hline & & $\mathrm{Z} 13$ & Succinate $(5 \mathrm{~g} / \mathrm{L})$ & & 0.511 \\
\hline & & Z15 & Ethanol $(5 \mathrm{~g} / \mathrm{L})$ & & 0.551 \\
\hline & & $\mathrm{Z} 17$ & Glycerol $(5 \mathrm{~g} / \mathrm{L})$ & & 0.643 \\
\hline \multirow{8}{*}{$\begin{array}{l}\text { Third } \\
7 / 16\end{array}$} & \multirow{8}{*}{$1-2$ drops in $50 \mathrm{~mL}$} & $\mathrm{Z} 19$ & Pyruvate (5 g/L) & \multirow{8}{*}{$\begin{array}{l}\text { Naphtha } \\
(\mathrm{H}, 5 \%)\end{array}$} & 0.428 \\
\hline & & $\mathbf{Z} 21$ & Glucose $(5 \mathrm{~g} / \mathrm{L})$ & & 0.678 \\
\hline & & $\mathrm{Z} 23$ & Fructose $(5 \mathrm{~g} / \mathrm{L})$ & & 0.605 \\
\hline & & $\mathrm{Z} 25$ & Lactate $(5 \mathrm{~g} / \mathrm{L})$ & & 1.040 \\
\hline & & $\mathrm{Z} 27$ & Succinate $(5 g / L)$ & & 0.716 \\
\hline & & Z29 & Glycerol $(5 g / L)$ & & 0.442 \\
\hline & & $\mathrm{Z31}$ & Acetate $(5 \mathrm{~g} / \mathrm{L})$ & & 0.010 \\
\hline & & $\mathrm{Z} 33$ & Ethanol $(5 \mathrm{~g} / \mathrm{L})$ & & 0.007 \\
\hline \multirow{7}{*}{$\begin{array}{l}\text { Fourth } \\
7 / 19\end{array}$} & \multirow{7}{*}{1.2 drops in $50 \mathrm{~mL}$} & $\mathrm{Z} 35$ & None & \multirow{7}{*}{$\begin{array}{l}\text { Naphtha } \\
(L, 5 \%)\end{array}$} & 0.011 \\
\hline & & $\mathrm{Z} 37$ & Pyruvate $(5 \mathrm{~g} / \mathrm{L})$ & & 0.115 \\
\hline & & Z39 & Glucose $(5 \mathrm{~g} / \mathrm{L})$ & & 0.178 \\
\hline & & $\mathrm{Z} 41$ & Fructose $(5 \mathrm{~g} / \mathrm{L})$ & & 0.010 \\
\hline & & $\mathrm{Z} 43$ & Lactate $(5 \mathrm{~g} / \mathrm{L})$ & & 0.292 \\
\hline & & $\mathrm{Z} 45$ & Succinate $(5 \mathrm{~g} / \mathrm{L})$ & & 0.008 \\
\hline & & $\mathrm{Z} 47$ & Glyceroi $(5 \mathrm{~g} / \mathrm{L})$ & & 0.012 \\
\hline \multirow[b]{4}{*}{$\begin{array}{l}\text { Fourth } \\
7 / 19\end{array}$} & \multirow[b]{4}{*}{1.2 drops in $50 \mathrm{~mL}$} & Da1 & None & \multirow[b]{4}{*}{$\begin{array}{l}\text { Naphtha } \\
(\mathrm{H}, 5 \%)\end{array}$} & 0.012 \\
\hline & & Da2 & Pyruvate $(5 \mathrm{~g} / \mathrm{L})$ & & 0.376 \\
\hline & & Da3 & Glucose $(5 \mathrm{~g} / \mathrm{L})$ & & 0.308 \\
\hline & & Da4 & Fructose $(5 \mathrm{~g} / \mathrm{L})$ & & 0.462 \\
\hline
\end{tabular}




\begin{tabular}{|c|c|c|c|c|c|}
\hline & & Da5 & Lactate $(5 g / L)$ & & 0.471 \\
\hline & & Da6 & Succinate $(5 \mathrm{~g} / \mathrm{L})$ & & 0.625 \\
\hline & & $\mathrm{Da} 7$ & Glycerol $(5 \mathrm{~g} / \mathrm{L})$ & & 0.389 \\
\hline \multirow{7}{*}{$\begin{array}{l}\text { Fifth } \\
7 / 20\end{array}$} & \multirow{7}{*}{$1-2$ drops in $50 \mathrm{~mL}$} & L1 & Glucose $(5 \mathrm{~g} / \mathrm{L})$ & \multirow{7}{*}{ None } & 0.113 \\
\hline & & L2 & Succinate $(5 \mathrm{~g} / \mathrm{L})$ & & 0.123 \\
\hline & & $\mathbf{L 3}$ & Glycerol $(5 \mathrm{~g} / \mathrm{L})$ & & 0.095 \\
\hline & & LA & Lactate $(5 \mathrm{~g} / \mathrm{L})$ & & 0.099 \\
\hline & & L5 & Acetate $(5 g / L)$ & & 0.065 \\
\hline & & L6 & Fructose $(5 \mathrm{~g} / \mathrm{L})$ & & 0.095 \\
\hline & & L7 & Pyruvate $(5 \mathrm{~g} / \mathrm{L})$ & & 0.331 \\
\hline \multirow{7}{*}{$\begin{array}{l}\text { Fifth } \\
7 / 21\end{array}$} & \multirow{7}{*}{$1-2$ drops in $50 \mathrm{~mL}$} & L8 & Glucose $(5 \mathrm{~g} / \mathrm{L})$ & \multirow{7}{*}{$\begin{array}{l}\text { None- } \\
\text { Millipore } \\
\text { Water }\end{array}$} & 0.289 \\
\hline & & L9 & Succinate $(5 \mathrm{~g} / \mathrm{L})$ & & 0.101 \\
\hline & & L10 & Glycerol $(5 \mathrm{~g} / \mathrm{L})$ & & 0.078 \\
\hline & & L11 & Lactate $(5 \mathrm{~g} / \mathrm{L})$ & & 0.059 \\
\hline & & L12 & Acetate $(5 \mathrm{~g} / \mathrm{L})$ & & 0.037 \\
\hline & & $\mathrm{L} 13$ & Fructose $(5 \mathrm{~g} / \mathrm{L})$ & & 0.324 \\
\hline & & L14 & Pyruvate $(5 \mathrm{~g} / \mathrm{L})$ & & 0.137 \\
\hline \multirow{6}{*}{$\begin{array}{l}\text { Sixth } \\
7 / 23\end{array}$} & \multirow{6}{*}{$1-2$ drops in $50 \mathrm{~mL}$} & $\mathrm{C} 1$ & Lactate $(5 \mathrm{~g} / \mathrm{L})$ & None & 0.223 \\
\hline & & $\mathrm{C} 2$ & Lactate $(5 \mathrm{~g} / \mathrm{L})$ & $\begin{array}{l}\text { Naphtha } \\
(\mathrm{H}, 5 \%) \\
\end{array}$ & 0.504 \\
\hline & & $\mathrm{C} 3$ & Lactate $(5 \mathrm{~g} / \mathrm{L})$ & $\begin{array}{l}\text { Naphtha } \\
(\mathrm{L}, 5 \%)\end{array}$ & 0.085 \\
\hline & & $\mathrm{C4}$ & Glycerol $(5 \mathrm{~g} / \mathrm{L})$ & None & 0.221 \\
\hline & & $\mathrm{C} 5$ & Glycerol (5 g/L) & $\begin{array}{l}\text { Naphtha } \\
(\mathrm{H}, 5 \%) \\
\end{array}$ & 0.286 \\
\hline & & $\mathrm{C} 6$ & Glycerol $(5 \mathrm{~g} / \mathrm{L})$ & $\begin{array}{l}\text { Naphtha } \\
(\mathrm{L}, 5 \%)\end{array}$ & 0.189 \\
\hline \multirow{9}{*}{$\begin{array}{l}\text { Seventh } \\
7 / 26\end{array}$} & \multirow{7}{*}{$1-2$ drops in $50 \mathrm{~mL}$} & L15 & Lactate $(5 \mathrm{~g} / \mathrm{L})$ & \multirow{3}{*}{ None } & 0.066 \\
\hline & & L16 & Lactate $(5 \mathrm{~g} / \mathrm{L})$ & & 0.041 \\
\hline & & $\mathrm{L} 17$ & Lactate $(5 \mathrm{~g} / \mathrm{L})$ & & 0.067 \\
\hline & & L18 & Lactate $(5 \mathrm{~g} / \mathrm{L})$ & \multirow{6}{*}{$\begin{array}{l}\text { Naphtha } \\
(\mathrm{H}, 5 \%)\end{array}$} & 0.410 \\
\hline & & L19 & Lactate $(5 \mathrm{~g} / \mathrm{L})$ & & 0.560 \\
\hline & & $\mathrm{L} 20$ & Lactate $(5 \mathrm{~g} / \mathrm{L})$ & & 0.510 \\
\hline & & L21 & Lactate $(5 \mathrm{~g} / \mathrm{L})$ & & - \\
\hline & \multirow{2}{*}{ No Bacteria } & $\mathrm{L} 22$ & Lactate $(5 \mathrm{~g} / \mathrm{L})$ & & - \\
\hline & & $\mathrm{L} 23$ & Lactate $(5 \mathrm{~g} / \mathrm{L})$ & & - \\
\hline \multirow{2}{*}{$\begin{array}{l}\text { Seventh } \\
7 / 28\end{array}$} & \multirow{2}{*}{$\begin{array}{c}1 \mathrm{~mL} \text { of culture from } \\
\mathrm{C} 3 \text { and } \mathrm{C} 6\end{array}$} & $\mathrm{Da} 8$ & Lactate $(5 \mathrm{~g} / \mathrm{L})$ & \multirow{2}{*}{$\begin{array}{l}\text { Naphtha } \\
(\mathrm{L}, 5 \%)\end{array}$} & - \\
\hline & & $\mathrm{Da} 9$ & Glycerol $(5 \mathrm{~g} / \mathrm{L})$ & & - \\
\hline
\end{tabular}




\begin{tabular}{|c|c|c|c|c|c|}
\hline \multirow{6}{*}{$\begin{array}{l}\text { Eighth } \\
7 / 30\end{array}$} & $0.5 \mathrm{~mL}$ in $300 \mathrm{~mL}$ & $\mathbf{Z}(1)$ & Lactate $(5 \mathrm{~g} / \mathrm{L})$ & \multirow{2}{*}{$\begin{array}{l}\text { Naphtha } \\
(\mathrm{H}, 2 \%)\end{array}$} & 0.190 \\
\hline & None & $Z(2)$ & Lactate $(5 \mathrm{~g} / \mathrm{L})$ & & 0.090 \\
\hline & $0.5 \mathrm{~mL}$ in $300 \mathrm{~mL}$ & $Z(3)$ & Lactate $(5 \mathrm{~g} / \mathrm{L})$ & None & 0.027 \\
\hline & $0.5 \mathrm{~mL}$ in $300 \mathrm{~mL}$ & $Z(4)$ & Lactate $(5 \mathrm{~g} / \mathrm{L})$ & Extracted & 0.153 \\
\hline & None & $\mathrm{Z}(5)$ & Lactate $(5 \mathrm{~g} / \mathrm{L})$ & $(\mathrm{H}, 2 \%)$ & 0.085 \\
\hline & $0.5 \mathrm{~mL}$ in $300 \mathrm{~mL}$ & $\mathrm{Z}(6)$ & Lactate $(5 \mathrm{~g} / \mathrm{L})$ & None & 0.038 \\
\hline \multirow{4}{*}{$\begin{array}{l}\text { Eighth } \\
8 / 2\end{array}$} & \multirow{4}{*}{$1-2$ drops in $50 \mathrm{~mL}$} & $\mathrm{DC1}$ & Lactate $(5 \mathrm{~g} / \mathrm{L})$ & \multirow{2}{*}{$\begin{array}{c}\text { Water } \\
\text { extracted } \\
\text { from } \\
\text { naphtha } \\
(\mathrm{H}, 5 \%)\end{array}$} & 0.460 \\
\hline & & $\mathrm{DC} 2$ & Lactate $(5 \mathrm{~g} / \mathrm{L})$ & & 0.433 \\
\hline & & $\mathrm{DC3}$ & Lactate $(5 \mathrm{~g} / \mathrm{L})$ & \multirow[t]{2}{*}{ None } & 0.019 \\
\hline & & $\mathrm{DC4}$ & Lactate $(5 \mathrm{~g} / \mathrm{L})$ & & 0.012 \\
\hline \multirow{8}{*}{$\begin{array}{l}\text { Eighth } \\
8 / 3\end{array}$} & \multirow{8}{*}{$\begin{array}{l}1 \mathrm{~mL} \text { from } \mathrm{C} 3 \text { and } \mathrm{C} 6 \\
\text { in } 50 \mathrm{~mL}\end{array}$} & LB1 & & & \\
\hline & & LB2 & & & \\
\hline & & LB3 & & & \\
\hline & & LB4 & & & \\
\hline & & LB5 & & & \\
\hline & & LB6 & & & \\
\hline & & LB7 & & & \\
\hline & & LB8 & Lactate $(5 \mathrm{~g} / \mathrm{L})$ & & 0.027 \\
\hline
\end{tabular}


Table 2. Enrichment of Denitrifying Bacteria on Various Carbon Sources Using Heavy Straight Run Naphtha as the Sulfur Source

\begin{tabular}{|c|c|c|c|c|c|}
\hline Transfer & Amount of Inoculum & Bottle & Carbon Source & $\begin{array}{l}\text { Sulfur } \\
\text { Source }\end{array}$ & $\begin{array}{l}\text { Growth } \\
600 \mathrm{~nm}\end{array}$ \\
\hline $\begin{array}{l}\text { First } \\
7 / 23\end{array}$ & $\begin{array}{c}2 \mathrm{~mL} \text { of mixture } \\
\text { of sludge, soil and } \\
\text { petroleum waste } \\
\text { samples }\end{array}$ & Mix 3 & $\begin{array}{l}\text { Acetate, Pyruvate, } \\
\text { Glucose, Fructose, } \\
\text { Methanol, Lactate, } \\
\text { Succinate, Ethanol, } \\
\text { Glycerol-1 } \mathrm{g} / \mathrm{L} \text { each }\end{array}$ & $\begin{array}{l}\text { Naptha } \\
(\mathrm{L}, 5 \%)\end{array}$ & 0.437 \\
\hline \multirow{9}{*}{$\begin{array}{l}\text { Second } \\
7 / 26\end{array}$} & \multirow{9}{*}{$\begin{array}{l}1.2 \text { drops from Mix } 3 \\
\text { in } 50 \mathrm{~mL}\end{array}$} & $\mathrm{ZJ} 1$ & Acetate $(5 g /)$ & & \\
\hline & & $\mathrm{ZJ} 2$ & Pyruvate $(5 \mathrm{~g} / \mathrm{L})$ & & \\
\hline & & $\mathrm{ZJ} 3$ & Glucose $(5 \mathrm{~g} / \mathrm{L})$ & & \\
\hline & & $\mathrm{ZJ} 4$ & Fructose $(5 \mathrm{~g} / \mathrm{L})$ & & \\
\hline & & ZJ5 & Methanol $(5 \mathrm{~g} / \mathrm{L})$ & & \\
\hline & & $\mathrm{ZJ} 6$ & Ethanol $(5 \mathrm{~g} / \mathrm{L})$ & & \\
\hline & & $\mathrm{ZJ} 7$ & Lactate $(5 \mathrm{~g} / \mathrm{L})$ & & \\
\hline & & ZJ8 & Succinate $(5 \mathrm{~g} / \mathrm{L})$ & & \\
\hline & & ZJ9 & Glycerol $(5 \mathrm{~g} / \mathrm{L})$ & & \\
\hline \multirow{4}{*}{$\begin{array}{l}\text { First } \\
8 / 3\end{array}$} & $1 \mathrm{~mL}$ of sludge & LA1 & \multirow{4}{*}{$\begin{array}{c}\text { Pyruvate, Glucose } \\
\text { Fructose, Succinate } \\
\text { Glycerol, Acetate, } \\
\text { Lactate } \\
1 \mathrm{~g} / \mathrm{L} \text { each }\end{array}$} & \multirow{4}{*}{$\begin{array}{l}\text { Naphtha } \\
\text { (L, 5\%) }\end{array}$} & \\
\hline & $1 \mathrm{~mL}$ of pet. waste & LA2 & & & \\
\hline & $1 \mathrm{~mL}$ of sludge $37^{\circ} \mathrm{C}$ & LA3 & & & \\
\hline & $\begin{array}{c}1 \mathrm{~mL} \text { of pet. waste } \\
37^{\circ} \mathrm{C}\end{array}$ & LA4 & & & \\
\hline
\end{tabular}

Table 3. Enrichment of Denitrifying Bacteria which on Various Carbon Sources Using Water Extracted Naphtha as the Sulfur Source

\begin{tabular}{|c|c|c|c|c|c|}
\hline Transfer & Amount of Inoculum & Bottle & Carbon Source & $\begin{array}{l}\text { Sulfur } \\
\text { Source }\end{array}$ & $\begin{array}{l}\text { Growth } \\
600 \mathrm{~nm}\end{array}$ \\
\hline $\begin{array}{l}\text { First } \\
7 / 30\end{array}$ & Mixed sludge & $Z(7)$ & $\begin{array}{l}\text { Acetate, Pyruvate, } \\
\text { Glucose, Fructose, } \\
\text { Lactate, Succinate, } \\
\text { Glycerol-1 g/L each }\end{array}$ & \multirow{9}{*}{$\begin{array}{c}\text { Extracted } \\
\text { Naphtha } \\
\text { (H, } 5 \%) \\
\text { through } \\
\text { water }\end{array}$} & 0.456 \\
\hline \multirow{8}{*}{$\begin{array}{l}\text { Second } \\
8 / 4\end{array}$} & \multirow{8}{*}{$\begin{array}{c}0.5 \mathrm{~mL} \text { from } \mathrm{Z}(7) \text { in } \\
50 \mathrm{~mL}\end{array}$} & $\mathrm{Z}(8)$ & Pyruvate $(5 \mathrm{~g} / \mathrm{L})$ & & \\
\hline & & $\mathrm{Z}(9)$ & Pyruvate $(5 g / L)$ & & \\
\hline & & $\mathrm{Z}(10)$ & Acetate $(5 \mathrm{~g} / \mathrm{L})$ & & \\
\hline & & $Z(11)$ & Acetate $(5 \mathrm{~g} / \mathrm{L})$ & & \\
\hline & & $\mathrm{Z}(12)$ & Glycerol $(5 \mathrm{~g} / \mathrm{L})$ & & \\
\hline & & $Z(13)$ & Glycerol $(5 \mathrm{~g} / \mathrm{L})$ & & \\
\hline & & $Z(14)$ & Lactate $(5 \mathrm{~g} / \mathrm{L})$ & & \\
\hline & & $Z(15)$ & Lactate $(5 \mathrm{~g} / \mathrm{L})$ & & \\
\hline
\end{tabular}


Table 4. Enrichment of Denitrifying Bacteria on Various Carbon Sources Using Coal Liquid $E$ as the Sulfur Source

\begin{tabular}{|c|c|c|c|c|c|}
\hline Transfer & Amount of Inoculum & Bottle & Carbon Source & $\begin{array}{l}\text { Sulfur } \\
\text { Source }\end{array}$ & Growth \\
\hline $\begin{array}{l}\text { First } \\
7 / 9\end{array}$ & $\begin{array}{l}5 \mathrm{~mL} \text { of mixture of } \\
\text { sludge, soil and } \\
\text { petroleum waste } \\
\text { samples }\end{array}$ & B & $\begin{array}{l}\text { Acetate, Pyruvate, } \\
\text { Glucose, Fructose, } \\
\text { Methanol, Lactate, } \\
\text { Succinate, Ethanol, } \\
\text { Glycerol-1 g/L each }\end{array}$ & \multirow{23}{*}{$\begin{array}{l}\text { Coal } \\
\text { Liquid } \\
\mathrm{E} \\
(5 \%)\end{array}$} & ++ \\
\hline \multirow{9}{*}{$\begin{array}{c}\text { Second } \\
7 / 12\end{array}$} & \multirow{9}{*}{$1-2$ drops in $50 \mathrm{~mL}$} & $\mathrm{Z} 2$ & Acetate $(5 \mathrm{~g} / \mathrm{L})$ & & - \\
\hline & & $\mathrm{Z4}$ & Pyruvate $(5 \mathrm{~g} / \mathrm{L})$ & & ++ \\
\hline & & $\mathrm{Z6}$ & Glucose $(5 \mathrm{~g} / \mathrm{L})$ & & ++ \\
\hline & & $\mathrm{Z8}$ & Fructose $(5 \mathrm{~g} / \mathrm{L})$ & & ++ \\
\hline & & $\mathrm{Z} 10$ & Methanol $(5 \mathrm{~g} / \mathrm{L})$ & & - \\
\hline & & $\mathrm{Z} 12$ & Lactate $(5 \mathrm{~g} / \mathrm{L})$ & & - \\
\hline & & $\mathrm{Z} 14$ & Succinate $(5 \mathrm{~g} / \mathrm{L})$ & & - \\
\hline & & $\mathrm{Z} 16$ & Ethanol $(5 \mathrm{~g} / \mathrm{L})$ & & - \\
\hline & & $\mathrm{Z} 18$ & Glycerol $(5 \mathrm{~g} / \mathrm{L})$ & & ++ \\
\hline \multirow{4}{*}{$\begin{array}{l}\text { Third } \\
7 / 16\end{array}$} & \multirow{4}{*}{$1-2$ drops in $50 \mathrm{~mL}$} & $\mathrm{Z} 20$ & Pyruvate $(5 \mathrm{~g} / \mathrm{L})$ & & ++ \\
\hline & & $\mathrm{Z} 22$ & Glucose $(5 \mathrm{~g} / \mathrm{L})$ & & - \\
\hline & & $\mathrm{Z} 24$ & Fructose $(5 \mathrm{~g} / \mathrm{L})$ & & ++ \\
\hline & & $\mathrm{Z} 26$ & Glycerol $(5 \mathrm{~g} / \mathrm{L})$ & & \\
\hline \multirow{2}{*}{$\begin{array}{c}\text { Fourth } \\
7 / 19\end{array}$} & \multirow[t]{2}{*}{$1-2$ drops in $50 \mathrm{~mL}$} & $\mathrm{Z} 28$ & Glycerol $(5 \mathrm{~g} / \mathrm{L})$ & & ++ \\
\hline & & $\mathrm{Z30}$ & Glycerol $(5 \mathrm{~g} / \mathrm{L})$ & & ++ \\
\hline \multirow{2}{*}{$\begin{array}{l}\text { Fourth } \\
7 / 23\end{array}$} & \multirow[t]{2}{*}{$1-2$ drops in $50 \mathrm{~mL}$} & $\mathrm{Z} 32$ & Pyruvate $(5 \mathrm{~g} / \mathrm{L})$ & & \\
\hline & & $\mathrm{Z} 34$ & Fructose $(5 \mathrm{~g} / \mathrm{L})$ & & \\
\hline Fifth, 7/23 & 1.2 drops in $50 \mathrm{~mL}$ & $\mathrm{Z} 36$ & Glycerol $(5 \mathrm{~g} / \mathrm{L})$ & & \\
\hline \multirow{2}{*}{$\begin{array}{l}\text { Fourth } \\
7 / 27\end{array}$} & \multirow[t]{2}{*}{$1-2$ drops in $50 \mathrm{~mL}$} & $\mathrm{Z38}$ & Pyruvate $(5 \mathrm{~g} / \mathrm{L})$ & & + \\
\hline & & $\mathrm{Z} 40$ & Fructose $(5 \mathrm{~g} / \mathrm{L})$ & & \\
\hline Fifth, $7 / 27$ & 1.2 drops in $50 \mathrm{~mL}$ & $\mathrm{Z} 42$ & Glycerol $(5 \mathrm{~g} / \mathrm{L})$ & & \\
\hline $\begin{array}{c}\text { First } \\
8 / 4\end{array}$ & $\begin{array}{l}\text { Mixture of sludge and } \\
\text { pet. waste samples }\end{array}$ & $\mathrm{D}$ & $\begin{array}{l}\text { Glucose, Glycerol, } \\
\text { Succinate, Pyruvate, } \\
\text { Acetate, Lactate, } \\
\text { Fructose-1 g/L each }\end{array}$ & & \\
\hline
\end{tabular}


Table 5. Sulfur Removal from Coal Liquid F (2\%) by Denitrifying Enrichments Using Acetate as the Carbon Source

\begin{tabular}{|c|c|c|c|c|c|c|c|c|}
\hline \multirow{2}{*}{$\begin{array}{c}\text { Time } \\
\text { (h) }\end{array}$} & \multicolumn{2}{|c|}{ A (control) } & \multicolumn{2}{|c|}{$\mathrm{B}$ (DT4) } & \multicolumn{2}{|c|}{$\mathrm{C}$ (DT8) } & \multicolumn{2}{|c|}{$\mathrm{D}$ (DT3) } \\
\hline & $\mathrm{S} \%$ & $\mathrm{pH}$ & S\% & $\mathrm{pH}$ & S\% & $\mathrm{pH}$ & S\% & $\mathrm{pH}$ \\
\hline 0 & 0.0528 & 8.05 & 0.0522 & 8.16 & 0.0559 & 8.24 & 0.0505 & 8.05 \\
\hline 24 & 0.0516 & 8.19 & 0.0503 & 8.28 & 0.0541 & 8.50 & 0.0540 & 8.19 \\
\hline 48 & 0.0550 & 8.34 & 0.0512 & 8.49 & 0.0523 & 8.54 & 0.0517 & 8.34 \\
\hline 72 & 0.0523 & 8.62 & 0.0516 & 8.53 & 0.0466 & 8.59 & 0.0499 & 8.62 \\
\hline 96 & 0.0538 & 8.61 & 0.0500 & 8.58 & 0.0494 & 8.64 & 0.0505 & 8.61 \\
\hline 120 & 0.0531 & 8.31 & 0.0501 & 8.71 & 0.0486 & 8.79 & 0.0518 & 8.31 \\
\hline 144 & & 8.34 & 0.0510 & 8.54 & 0.0540 & 8.67 & & \\
\hline 168 & 0.0498 & 8.50 & 0.0506 & 8.67 & 0.0513 & 8.63 & 0.0524 & 8.34 \\
\hline 192 & 0.0506 & 8.51 & 0.0509 & 8.69 & 0.0540 & 8.63 & 0.0531 & 8.50 \\
\hline 216 & 0.0506 & 8.61 & 0.0500 & 8.69 & 0.0558 & 8.71 & 0.0517 & 8.51 \\
\hline 264 & 0.0503 & 8.57 & 0.052 .6 & 8.58 & 0.0515 & 8.41 & 0.0506 & 8.61 \\
\hline 288 & 0.0529 & 8.34 & 0.0532 & 8.50 & 0.0539 & 7.63 & 0.0519 & 8.57 \\
\hline 312 & 0.0518 & 8.41 & 0.0481 & 8.52 & 0.0596 & 7.62 & 0.0525 & 8.34 \\
\hline
\end{tabular}

Table 6. Bacterial Utilization on Sulfur from Full Range Cracked Naphtha by Denitrifying Bacteria

\begin{tabular}{||l|c|c|c||}
\hline Inoculation & Carbon & Sulfur & OD $(600 \mathrm{~nm})$ \\
\hline \hline First $(8 / 15)$ & Lactate $(5 \mathrm{~g} / 1)$ & Naphtha $(\mathrm{H}, 10 \%)$ & 0.967 \\
\hline Second $(8 / 20)$ & Lactate $(5 \mathrm{~g} /)$ & 1st-treated naphtha & 0.786 \\
\hline Third $(8 / 23)$ & Lactate $(5 \mathrm{~g} / 1)$ & 2nd-treated naphtha & 0.465 \\
\hline Fourth $(8 / 27)$ & Lactate $(5 \mathrm{~g} /)$ & 3rd-treated naphtha & 0.367 \\
\hline Fifth $(9 / 1)$ & Lactate $(5 \mathrm{~g} / 1)$ & 4th-treated naphtha & 0.286 \\
\hline Sixth $(9 / 8)$ & Lactate $(5 \mathrm{~g} /)$ & 5th-treated naphtha & 0.612 \\
\hline Seventh $(9 / 17)$ & Lactate $(5 \mathrm{~g} / 1)$ & 6th-treated naphtha & 0.705 \\
\hline Eighth $(9 / 26)$ & Lactate $(5 \mathrm{~g} / 1)$ & 7th-treated naphtha & 0.721 \\
\hline
\end{tabular}

Table 7. Growth of Denitrifying Bacteria with Heavy Straight Run Naphtha as the Sole Sulfur Source.

\begin{tabular}{|l|c|c|c|}
\hline \multicolumn{1}{|c|}{ Inoculation } & Carbon Source & Sulfur Source & OD (600nm) \\
\hline First $(7 / 23)$ & Mixture of sugars & Naphtha (5\%) & 0.437 \\
\hline Second $(7 / 26)$ & Fructose $(5 \mathrm{~g} / \mathrm{L})$ & Naphtha $(5 \%)$ & 0.556 \\
\hline Third $(8 / 9)$ & Pyruvate $(5 \mathrm{~g} / \mathrm{L})$ & Naphtha $(5 \%)$ & 1.125 \\
\hline Fourth $(8 / 26)$ & Pyruvate $(5 \mathrm{~g} / \mathrm{L})$ & Naphtha $(5 \%)$ & 0.893 \\
\hline Fifth $(9 / 1)$ & Pyruvate $(5 \mathrm{~g} / \mathrm{L})$ & Naphtha $(5 \%)$ & \\
\hline
\end{tabular}


Table 8. Enrichment of Sulfate-Reducing Bacteria on Various Carbon Sources Using Naphtha as the Nitrogen Source

\begin{tabular}{|c|c|c|c|c|c|}
\hline Transfer & Amount of Inoculum & Bottle & Carbon Source & $\begin{array}{c}\text { Nitrogen } \\
\text { Source }\end{array}$ & $\begin{array}{l}\text { Growth } \\
600 \mathrm{~nm} \\
\end{array}$ \\
\hline $\begin{array}{l}\text { First } \\
7 / 13\end{array}$ & $\begin{array}{l}2 \mathrm{~mL} \text { of mixture of } \\
\text { sludge, soil and pet. } \\
\text { waste samples }\end{array}$ & Z19 & $\begin{array}{l}\text { Acetate, Pyruvate, } \\
\text { Glucose, Fructose, } \\
\text { Lactate, Succinate, } \\
\text { Glycerol-1 } g / L \text { each }\end{array}$ & $\begin{array}{l}\text { Naphtha } \\
(\mathrm{H}, 5 \%)\end{array}$ & 0.076 \\
\hline \multirow{2}{*}{$\begin{array}{l}\text { Second } \\
7 / 19\end{array}$} & \multirow[t]{2}{*}{ 1-2 drops in $50 \mathrm{~mL}$} & $\mathrm{D} 1$ & Glucose $(5 \mathrm{~g} / \mathrm{L})$ & \multirow{2}{*}{$\begin{array}{l}\text { Naphtha } \\
(\mathrm{H}, 5 \%)\end{array}$} & 0.170 \\
\hline & & D2 & Glycerol $(5 \mathrm{~g} / \mathrm{L})$ & & 0.008 \\
\hline \multirow{7}{*}{$\begin{array}{l}\text { Third } \\
7 / 23\end{array}$} & \multirow{7}{*}{ 1-2 drops in $50 \mathrm{~mL}$} & Db1 & Acetate $(5 \mathrm{~g} / \mathrm{L})$ & \multirow{7}{*}{$\begin{array}{l}\text { Naphtha } \\
(\mathrm{H}, 5 \%)\end{array}$} & 0.043 \\
\hline & & $\mathrm{Db} 2$ & Fructose $(5 \mathrm{~g} / \mathrm{L})$ & & 0.148 \\
\hline & & $\mathrm{Db} 3$ & Glucose $(5 \mathrm{~g} / \mathrm{L})$ & & 0.156 \\
\hline & & Db4 & Glycerol $(5 \mathrm{~g} / \mathrm{L})$ & & 0.115 \\
\hline & & Db5 & Lactate $(5 \mathrm{~g} / \mathrm{L})$ & & 0.036 \\
\hline & & Db6 & Pyruvate $(5 \mathrm{~g} / \mathrm{L})$ & & 0.120 \\
\hline & & $\mathrm{Db} 7$ & Succinate $(5 \mathrm{~g} / \mathrm{L})$ & & 0.032 \\
\hline \multirow{8}{*}{$\begin{array}{c}\text { Fourth } \\
7 / 27\end{array}$} & \multirow{8}{*}{$1-2$ drops in $50 \mathrm{~mL}$} & $\mathrm{~L} 24$ & Fructose $(5 \mathrm{~g} / \mathrm{L})$ & $\begin{array}{l}\text { Naphtha } \\
(\mathrm{H}, 5 \%)\end{array}$ & \\
\hline & & $\mathrm{L} 25$ & Fructose $(5 \mathrm{~g} / \mathrm{L})$ & None & \\
\hline & & L26 & Giucose $(5 \mathrm{~g} / \mathrm{L})$ & $\begin{array}{l}\text { Naphtha } \\
(\mathrm{H}, 5 \%) \\
\end{array}$ & \\
\hline & & $\mathrm{L} 27$ & Glucose $(5 \mathrm{~g} / \mathrm{L})$ & None & \\
\hline & & $\mathrm{L} 28$ & Glycerol $(5 \mathrm{~g} / \mathrm{L})$ & $\begin{array}{l}\text { Naphtha } \\
(\mathrm{H}, 5 \%) \\
\end{array}$ & \\
\hline & & $\mathrm{L} 29$ & Glycerol $(5 \mathrm{~g} / \mathrm{L})$ & None & \\
\hline & & $\mathrm{L} 30$ & Pyruvate $(5 \mathrm{~g} / \mathrm{L})$ & $\begin{array}{l}\text { Naphtha } \\
(\mathrm{H}, 5 \%) \\
\end{array}$ & \\
\hline & & L31 & Pyruvate $(5 \mathrm{~g} / \mathrm{L})$ & None & \\
\hline \multirow{4}{*}{$\begin{array}{c}\text { Fourth } \\
8 / 4\end{array}$} & \multirow{4}{*}{$0.5 \mathrm{~mL}$ in $50 \mathrm{~mL}$} & $\mathrm{Db} 8$ & Fructose $(5 \mathrm{~g} / \mathrm{L})$ & \multirow{4}{*}{$\begin{array}{l}\text { Naphtha } \\
(\mathrm{H}, 2 \%) \\
0.2 \mathrm{~mL} \\
\text { filtered } \\
\text { Vitamin B }\end{array}$} & \\
\hline & & Db9 & Glucose $(5 \mathrm{~g} / \mathrm{L})$ & & \\
\hline & & $\mathrm{Db} 10$ & Glycerol $(5 \mathrm{~g} / \mathrm{L})$ & & \\
\hline & & Db11 & Pyruvate $(5 \mathrm{~g} / \mathrm{L})$ & & \\
\hline
\end{tabular}


Table 9. Enrichment of Sulfate-Reducing Bacteria on Various Carbon Sources Using Coal Liquid $\mathbf{E}$ as the Nitrogen Source

\begin{tabular}{|c|c|c|c|c|c|}
\hline Transfer & Amount of Inoculum & Bottle & Carbon Source & $\begin{array}{c}\text { Nitrogen } \\
\text { Source }\end{array}$ & $\begin{array}{l}\text { Growth } \\
600 \mathrm{~nm}\end{array}$ \\
\hline $\begin{array}{l}\text { First } \\
7 / 13\end{array}$ & $\begin{array}{l}2 \mathrm{~mL} \text { of mixture of } \\
\text { sludge, soil and pet. } \\
\text { waste samples }\end{array}$ & $\mathbf{Z 2 0}$ & $\begin{array}{l}\text { Acetate, Pyruvate, } \\
\text { Glucose, Fructose, } \\
\text { Lactate, Succinate, } \\
\text { Glycerol-1 g/L each }\end{array}$ & $\begin{array}{c}\text { Coal } \\
\text { Liquid } \mathrm{E} \\
5 \%\end{array}$ & 0.042 \\
\hline \multirow{7}{*}{$\begin{array}{c}\text { Second } \\
7 / 23\end{array}$} & \multirow{7}{*}{$1-2$ drops in $50 \mathrm{~mL}$} & $\mathrm{ZC1}$ & Pyruvate $(5 \mathrm{~g} / \mathrm{L})$ & \multirow{7}{*}{$\begin{array}{l}\text { Coal } \\
\text { Liquid E } \\
5 \%\end{array}$} & \\
\hline & & $\mathrm{ZC2}$ & Glucose $(5 \mathrm{~g} / \mathrm{L})$ & & \\
\hline & & $\mathrm{ZC3}$ & Succinate $(5 \mathrm{~g} / \mathrm{L})$ & & \\
\hline & & $\mathrm{ZC4}$ & Acetate $(5 \mathrm{~g} / \mathrm{L})$ & & \\
\hline & & ZC5 & Glycerol $(5 \mathrm{~g} / \mathrm{L})$ & & \\
\hline & & ZC6 & Lactate $(5 \mathrm{~g} / \mathrm{L})$ & & \\
\hline & & $\mathrm{ZC7}$ & Fructose $(5 \mathrm{~g} / \mathrm{L})$ & & \\
\hline
\end{tabular}

Table 10. The Use of Sulfate Reducing Bacteria to Remove

Nitrogen from Coal Liquids (Anaerobic, $\mathrm{HCl}$ Pretreatment)

\begin{tabular}{|c|c|c|c|}
\hline Bottle No. & Source & Carbon & Growth \\
\hline \hline CB1 & Sludge & Lactate $(5 \mathrm{~g} /)$ & None \\
\hline CB2 & Sludge & Pyruvate $(5 \mathrm{~g} /)$ & None \\
\hline CB3 & Sludge & Succinate $(5 \mathrm{~g} /)$ & None \\
\hline CB4 & Sludge & Glucose $(5 \mathrm{~g} /)$ & None \\
\hline
\end{tabular}

Table 11. The Use of Sulfate Reducing Bacteria to Remove Nitrogen from Coal Liquids (Aerobic, $\mathrm{HCl}$ Pretreatment)

\begin{tabular}{|c|c|c|c|}
\hline Bottle No. & Source & Carbon & Growth \\
\hline \hline CB5 & Sludge & Glucose $(5 \mathrm{~g} /)$ & None \\
\hline CB6 & Sludge & Fructose $(5 \mathrm{~g} /)$ & None \\
\hline CB7 & Murphy's & Glucose $(5 \mathrm{~g} /)$ & None \\
\hline CB8 & Murphy's & Fructose $(5 \mathrm{~g} /)$ & None \\
\hline CB9 & Murphy's & Succinate $(5 \mathrm{~g} /)$ & None \\
\hline
\end{tabular}




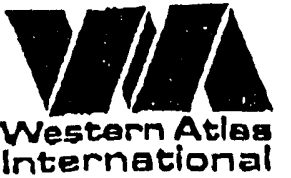
international

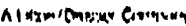

CORE LABDAATORIES

\section{LABORATORY TESTS RESULIS $06 / 24 / 93$}

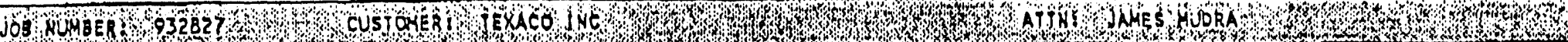

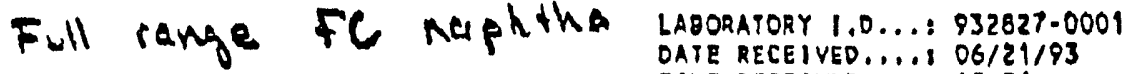

DATE SAMPLED........

TIME SAMPLED.......: :

WORK OESCRIPIIOK...: 291-92-0627-000

Total $S=0,1499$ wit REMRRS............

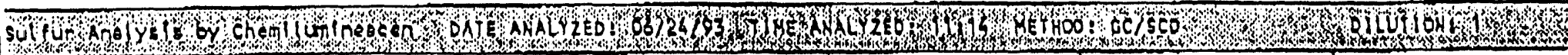

\section{TEST DESCRIPTION}

Hydrogen sulfide

Carbonyl sulfide

sulfur blexide

Carbon Dlsulfide

Methyl Mercopion

Ethyl Mercopton

lsopropyl Mercoptan

n-propyl Mercapion

tert-Butyl mercaptan

sec-Butyl mercapten

Isobueyl Kereootan

n-Butyl Mereopeon

Methyl sulfide

Ethyl Kethyl sulfide

Ethyl sulflide

Mothyl Disulfido

Ethyl Methyl Olsulfide

Ethyl Disulfide

Thlophane

Tatra-Hydro Thiophene

2-Methyl Thlophene

3-Methyl Thiophene

2-Ethyl Thiophene

3-Ethyl Thlophene

Benzothiophene

Methyl benzothlophenes

olmethyl benzothlophenes

Unidentifled sulfur Compounds

\begin{tabular}{|c|c|}
\hline FIKAL TEST RE & RESULT \\
\hline $\begin{array}{r}49 \\
2 \\
41 \\
91 \\
49 \\
1 \\
1 \\
1 \\
41 \\
19 \\
91 \\
41 \\
41 \\
49 \\
49 \\
9 \\
1 \\
57 \\
16 \\
75 \\
101 \\
39 \\
63 \\
273 \\
359 \\
82 \\
510\end{array}$ & . \\
\hline
\end{tabular}

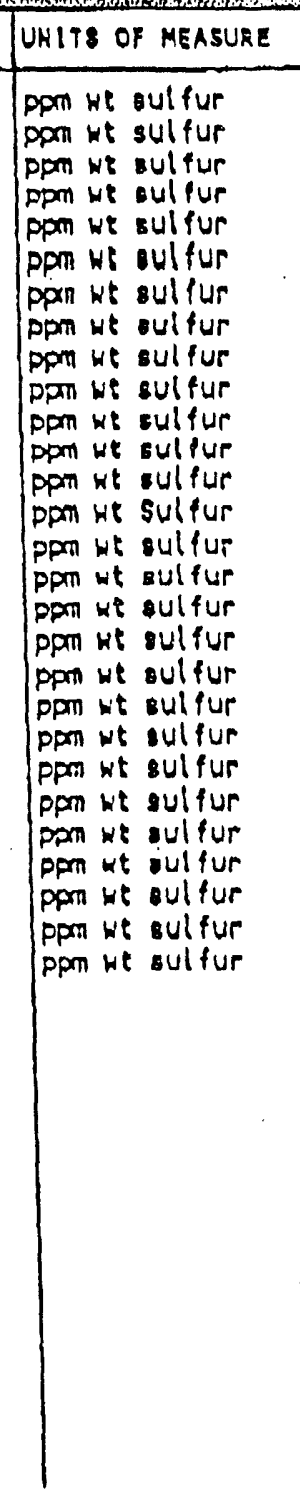

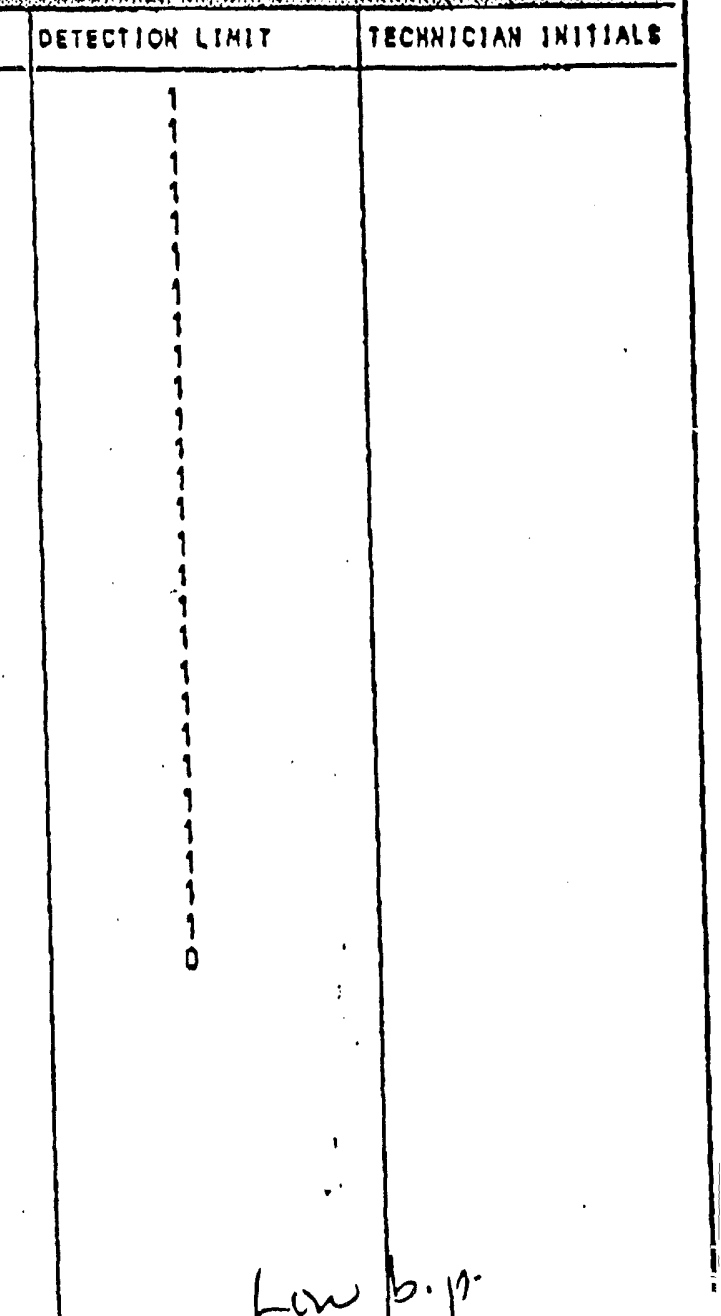




\section{CORE LABDRATORIES}

\section{LABORATORY TESTS RESULTS}

$\therefore \quad 06 / 24 / 93$

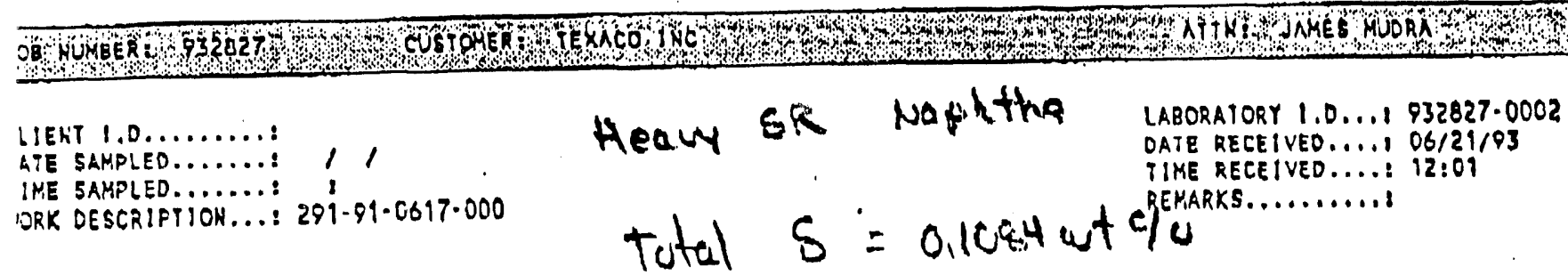

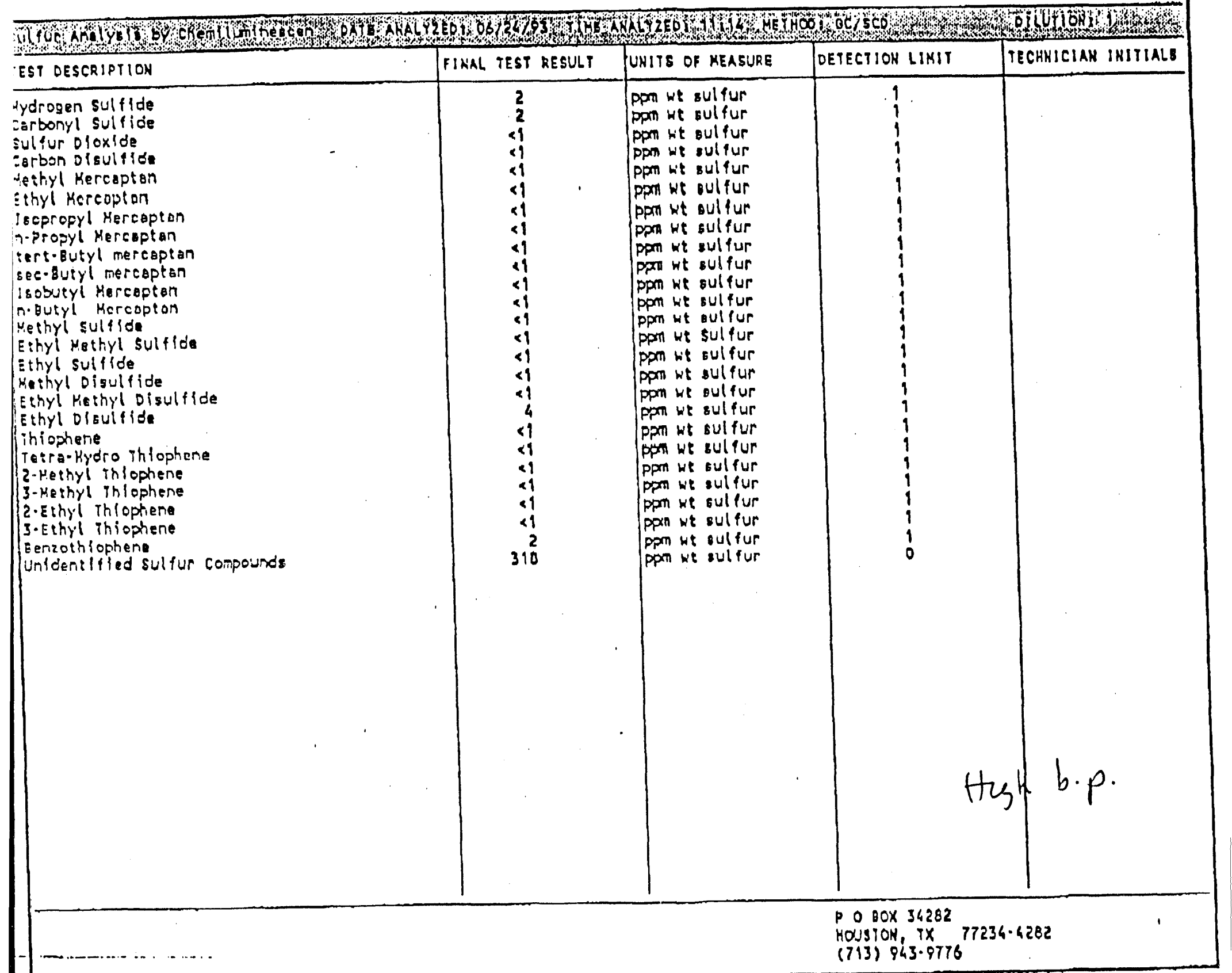




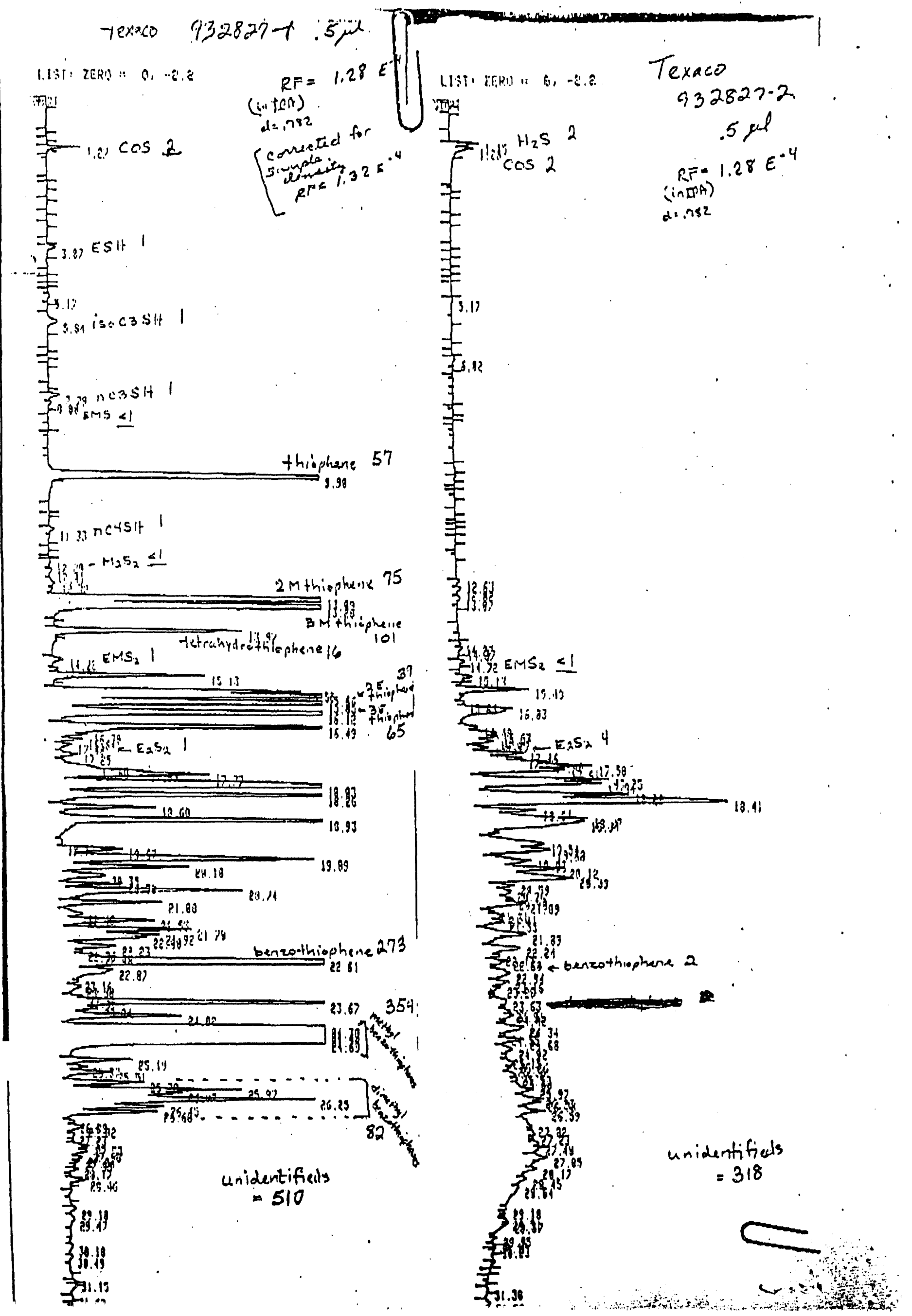



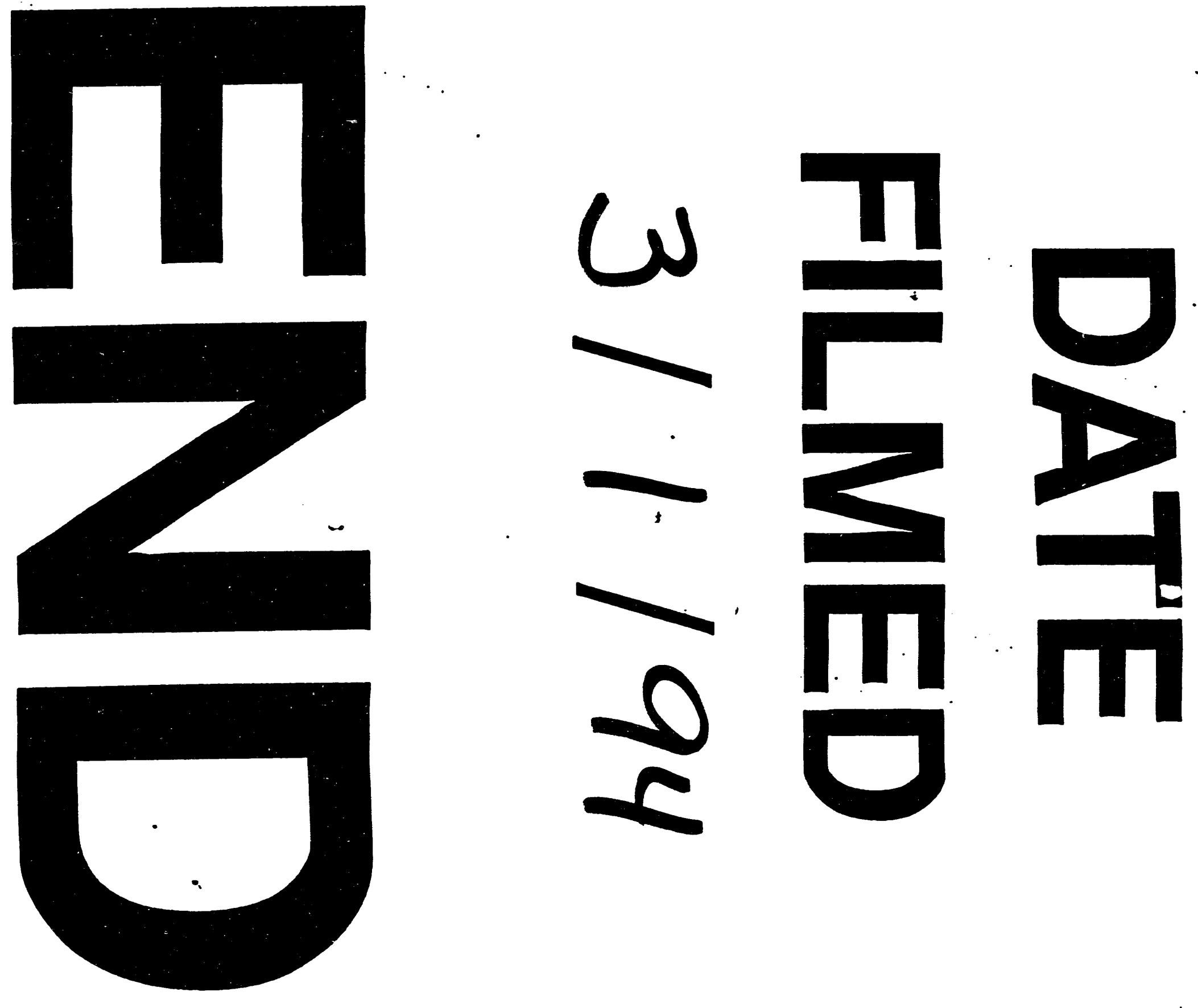
